\title{
Preparation and Evaluation of PEGylated and Folate-PEGylated Liposomes Containing Paclitaxel for Lymphatic Delivery
}

\author{
Hea-Young Cho, ${ }^{1}$ Chong Ki Lee, ${ }^{2}$ and Yong-Bok Lee ${ }^{3}$ \\ ${ }^{1}$ College of Pharmacy, CHA University, Seongnam-si 463-712, Republic of Korea \\ ${ }^{2}$ Department of Medical Management, Chodang University, Muan 534-701, Republic of Korea \\ ${ }^{3}$ College of Pharmacy, Chonnam National University, Gwangju 500-757, Republic of Korea \\ Correspondence should be addressed to Yong-Bok Lee; leeyb@chonnam.ac.kr
}

Received 19 September 2014; Accepted 24 November 2014

Academic Editor: Sabine Szunerits

Copyright (C) 2015 Hea-Young Cho et al. This is an open access article distributed under the Creative Commons Attribution License, which permits unrestricted use, distribution, and reproduction in any medium, provided the original work is properly cited.

\begin{abstract}
This study attempted to prepare polyethylene-glycol modified (PEGylated) and folate-PEGylated liposomes containing paclitaxel (Ptx) in order to reduce the toxicity and improve the bioavailability and biocompatibility by targeting drugs to the lymphatics using cancer cell specific ligand folate to prevent metastasis via the lymphatic system. Liposomes were prepared by lipid film hydration method using PEG and folate-PEG as surface modifiers. The mean particle size and encapsulation efficiency of liposomes were $114 \pm 6.81 \mathrm{~nm}$ and $81 \pm 2.3 \%$ for PEGylated liposome and $122 \pm 4.87 \mathrm{~nm}$ and $88 \pm 2.0 \%$ for folate-PEGylated liposome, respectively. According to stability test, it could be confirmed that PEGylated and folate-PEGylated liposomes were stable for at least 5 days. After intravenous administration of the PEGylated and folate-PEGylated liposomes to rats, the $\mathrm{CL}_{t}$ (total clearance) and $t_{1 / 2}$ (half-life) were significantly different $(P<0.05)$ compared with those of PADEXOL Inj. In targeting efficiency, calculated as the concentration ratio of Ptx in lymph nodes and plasma, there was significant increase in targeting efficiency at lymph nodes $(P<0.05)$. From these results, we could conclude that the prepared Ptx-containing PEGylated and folate-PEGylated liposomes are good candidates for the targeted delivery of the drug to lymphatic system.
\end{abstract}

\section{Introduction}

Paclitaxel is an anticancer drug that has a diterpenoid pseudoalkaloid structure having the molecular formula $\mathrm{C}_{47} \mathrm{H}_{51} \mathrm{NO}_{14}$, corresponding to molecular weight of $853 \mathrm{Da}$ [1]. Its anticancer activity was detected in 1967 and its therapeutic efficacy also includes ovarian, breast, head, and neck cancer, small lung cancer, colon cancer, multiple myeloma, melanoma, and Kaposi's sarcoma. The target of Ptx is microtubules, which are polymeric intracellular structures composed of chains of the dimeric protein tubulin [2,3]. However, there are two limitations for clinical application of Ptx. One is its low bioavailability (BA). Ptx is the substrate of P-glycoprotein (P-gp) [4]. The presence of this phenotype results in a decreased accumulation of drug within the cancer cell, which occurs by an increase in P-gp that functions as a drug efflux pump [4,5]. Another limitation of Ptx is its difficulty in clinical administration. Ptx is highly hydrophobic (water solubility $\leq 0.5 \mathrm{mg} / \mathrm{L}$ ). An adjuvant consisting of Cremophor EL (polyoxyethylated castor oil) and dehydrated alcohol has to be used in the current clinical administration of Ptx, which causes serious side effects [6]. It has been believed that side effects caused by Cremophor EL include hypersensitivity reactions, nephrotoxicity, and neurotoxicity [7]. It was also reported that Cremophor EL has influence on the functions of endothelial and vascular muscle and causes vasodilation, laboured breathing, lethargy, and hypotension [8].

Various formulations to increase BA of Ptx and reduce these side effects of Cremophor EL were studied and developed. For example, liposome [9, 10], prodrug [11], microsphere $[12,13]$, nanosphere $[14,15]$, self-emulsifying drug delivery systems [16], nanoparticle [17, 18], nanosponge [19, $20]$, and combination with P-gp inhibitors $[5,21]$ were developed and evaluated. Among these formulations, nanoparticles (NPs) are stable, solid colloidal particles consisting of biodegradable polymer or lipid materials and range in size 
from 10 to $1,000 \mathrm{~nm}$. Drugs can be absorbed onto the particle surface and are entrapped inside the polymer/lipid or dissolved within the particle matrix $[22,23]$. Over the past few decades, there have been considerable interests in developing nanosized liposomes as potential and effective drug delivery carriers due to their ability to control drug release and delivery. They can effectively deliver the drug to target sites and thus increase the therapeutic benefit while minimizing side effects $[24,25]$. Moreover, surface-modified PEGylated liposomes have been found to be important in potential therapeutic applications as injectable colloidal systems for controlled drug release with long circulation in the blood $[26,27]$.

The folate receptor (FR) has been recognized as playing a potential physiological role in cellular folate uptake. FRs are overexpressed in cancer cells, especially ovarian cancers [28-31]. A number of FR-targeted therapeutic and imaging agents have been evaluated in preclinical studies including liposomal agents [32-36]. Liposomes are taken up by kappa B cells, which are highly concentrated in lymphatic fluid, specifically compared with nontargeted liposomes [37]. Given the high level of FR expression in ovarian carcinomas and the effectiveness of Ptx as a first-line agent for this type of cancer and preventing its metastasis via the lymphatic system, there is good reason to develop an FR-targeted formulation for Ptx.

Many methods have been used in the preparation of liposomes. Among them, we selected the thin lipid film hydration method with sonication and a modification [38] because of its simplicity and reproducibility. In this study, we prepared and characterized the PEGylated and folate-PEGylated liposomes containing Ptx using the thin lipid film hydration method with sonication to increase the time it circulated in the blood and lymphatic delivery. We also evaluated pharmacokinetic characteristics and lymphatic targeting efficiency of these formulations in rats and the results were compared to those of commercial product, PADEXOL Inj. for intravenous (i.v.) administration.

\section{Materials and Methods}

\subsection{Materials}

2.1.1. Reagents. Ptx was kindly supplied by Dong Wha Pharm. Co., Ltd. (Seoul, Korea). Glimepiride was kindly supplied by Sam Chun Dang Pharm. Co., Ltd. (Seoul, Korea). PADEXOL Inj. was formulated in Acephorol 330 as a solubilizer and kindly supplied by Shin Poong Pharm. Co., Ltd. (Seoul, Korea). Egg-phosphatidylcholine (EPC), cholesterol (CHOL), pyridine, dimethyl sulfoxide (DMSO), and folic acid were purchased from Sigma Chemical Co. (St. Louis, MO, USA). 1,2-Distearoyl-sn-glycero-3-phosphoethanolamine-N-[methoxy (polyethylene glycol)-2000] (DSPE-mPEG) and 1,2-distearoyl-sn-glycero-3-phosphoethanolamine-N-[amino(polyethylene glycol)-2000] (aminoPEG-DSPE) were purchased from Avanti Polar Lipids Inc. (Alabaster, Al, USA). N,N'-dicyclohexylcarbodiimide (DCC) was purchased from Fluka (Buchs, Switzerland). Heparin sodium $(25,000 \mathrm{IU} / 5 \mathrm{~mL}$, Green Cross, Seoul, Korea) and phosphate buffered saline (PBS, pH 7.4, Sigma
Chemical Co., St. Louis, MO, USA) were used. Acetonitrile (Fischer Scientific., Fair Lawn, NJ, USA) was used for HPLC assay. HPLC grade water was obtained from a Milli-Q water purification system (Millipore Co., Milford, MA, USA) and was used throughout the study. Dialysis tubing cellulose membrane of molecular weight cut-off (MWCO) 1,000 (Spectra/Por CE, Spectrum laboratories Inc., Rancho Domingues, CA, USA) was used for synthesis of folate-PEG and MWCO 12,000 (Sigma-Aldrich Co., St. Louis, MO, USA) was used for the in vitro release test. All other chemicals and solvents were of analytical grade or highest quality available.

2.1.2. Instruments. Homogenizer (IKA-WERKE, KGD-79219, $\mathrm{GmbH} \&$ Co., Staufen, Germany), tip-type ultrasonicator (US-50, Nihonseiji Kalsha Ltd., Danbury, CT, USA), and ultraspeed centrifuge (Centrikon T-2070, Kontron, Switzerland) were used for synthesizing folate-PEG and preparation of liposomes. Particle size analyzer (Autosizer Lo-C, Malvern Instruments, Worcesterahire, UK), zeta potential analyzer (ELS-8000, Otsuka Electronics, Osaka, Japan), FE-SEM (field emission-scanning electron microscopy, S-4700, Hitachi Ltd., Tokyo, Japan), and TEM (transmission electron microscopy, JEM-200 FXII, JEOL Ltd, Tokyo, Japan) were used for evaluation of prepared liposomes. HPLC system (Shimadzu Corp., Kyoto, Japan) consisted of pump (Model LC-10AD $v p$ ), degasser (Model DGU-12A), UV detector (Model SPD$10 \mathrm{~A} v p$ ), system controller (SCL-10AD $v p$ ), and Shim-Pack CLC-ODS $(\mathrm{M})$ 15CM column $(4.6 \times 150 \mathrm{~mm}$ analytical column, $5 \mu \mathrm{m}$ particle size).

2.1.3. Experimental Animal. Experimental animals were managed according to a protocol approved by the Ethical Review Committee on Experimental Animals of Chonnam National University, South Korea. Eight-week-old male Sprague-Dawley rats weighing 190 210 g were obtained from Dae Han Laboratory Animal Research Center Co., Ltd. (Daejeon, Korea). Animals were housed separately in cages in a ventilated animal room with controlled temperature $(19 \pm$ $1^{\circ} \mathrm{C}$ ) and relative humidity $(50 \pm 5 \%)$ and kept on 12 hours light/dark cycle. The rats weighing 240 280 g were used.

\subsection{Methods}

2.2.1. Synthesis of Folate-PEG-DSPE. Folate-PEG-DSPE was synthesized as previously reported $[36,37,39]$. In brief, $25 \mathrm{mg}$ of folic acid was dissolved in $1 \mathrm{~mL}$ of DMSO and $100 \mathrm{mg}$ of amino-PEG-DSPE dissolved in $0.5 \mathrm{~mL}$ of pyridine containing $32.5 \mathrm{mg}$ of DCC was added. The reaction mixture was left to stand at room temperature for 4 hours and pyridine was removed by rotary vacuum evaporation. Three milliliters of water was added to the reaction mixture and the insoluble materials were removed by centrifugation at $36,000 \mathrm{~g}$ for 15 minutes (Centrikon T-2070, Kontron, Switzerland). The supernatant was dialyzed in Spectra/Por CE against saline for 24 hours and then against water for 24 hours. The dialyzed product (folate-PEG-DSPE) was lyophilized and stored at $-20^{\circ} \mathrm{C}$ before use.

2.2.2. Preparation of PEGylated and Folate-PEGylated Liposomes. The PEGylated and folate-PEGylated liposomes 
containing Ptx were prepared with a modification of the thin lipid film hydration method [38]. In detail, a mixture of EPC, CHOL, DSPE-mPEG, folate-PEG-DSPE, and Ptx dissolved in chloroform : methanol $=2: 1(\mathrm{v} / \mathrm{v})$ was evaporated to dryness by rotary vacuum evaporator under reduced pressure at $45^{\circ} \mathrm{C}$. A thin film was deposited on the inner wall of a Quickfit $50 \mathrm{~mL}$ round bottom glass flask. The dried lipid film was dispersed by gentle rotation at room temperature in $\mathrm{pH}$ 7.4 PBS solution. The resulting liposome dispersion (about $20 \mathrm{mM}$ total lipid) was gently shaken and sonicated for 10 minutes. Liposomes were centrifuged for 15 minutes $\left(50,000 \mathrm{~g}, 15^{\circ} \mathrm{C}\right)$ in order to remove nonencapsulated Ptx and multilamellar vehicles (MLV). Prepared liposomes were filtered using a $450 \mathrm{~nm}$ polyvinylidenedifluoride (PVDF) filter to remove large liposomes. The final liposomes in $\mathrm{pH}$ 7.4 PBS solution were stored at $4^{\circ} \mathrm{C}$ until later use.

(1) Determination of Optimal Concentration of DSPE-mPEG. EPC : CHOL: Ptx was fixed to $20: 10: 1$ molar ratio. DSPEmPEG was added to this mixture at concentrations of $0,1,2,3$, and 4 molar ratio. The final lipid concentration was adjusted to $20 \mathrm{mM}$. Liposomes were prepared as previously described. The optimal concentration of DSPE-mPEG was determined from the results of particle size and drug encapsulation efficiency (EE) of prepared liposomes.

(2) Determination of Optimal Concentration of Folate-PEGDSPE. EPC: CHOL: Ptx was fixed to a $20: 10: 1$ molar ratio. Folate-PEG-DSPE was added to this mixture at concentrations of $0,1,2,3$, and 4 molar ratio. The final lipid concentration was adjusted to $20 \mathrm{mM}$. Liposomes were prepared as previously described. The optimal concentration of DSPEmPEG was determined from the results of particle size and EE of prepared liposome.

\subsubsection{Characterization of PEGylated and Folate-PEGylated Liposomes}

(1) Determination of Particle Size of Liposomes. The mean particle size and size distribution analysis of liposomes were performed by the dynamic light scattering (DLS) method at $25^{\circ} \mathrm{C}$ with a $90^{\circ}$ scattering angle for optimum detection. One milliliter of the liposome dispersions was diluted with $5 \mathrm{~mL}$ of filtered PBS ( $\mathrm{pH}$ 7.4) using a polycarbonate filter $(0.1 \mu \mathrm{m}$ pore size, Milipore, $\mathrm{UK})$ and the size of liposomes was measured immediately. The presented results (mean \pm S.D.) are representatives of five independent experiments.

(2) Determination of Drug Encapsulation Efficiency. Drug concentrations were determined by reversed-phase HPLC. One hundred microliters of PEGylated and folate-PEGylated liposomes were mixed with $0.9 \mathrm{~mL}$ of methanol and vortexed vigorously for 1 minute. The solution was then diluted with a specific volume of mobile phase and injected into HPLC. The chromatographic column was Shim-Pack CLC-ODS (M) $15 \mathrm{CM}$ reversed-phase column $(4.6 \times 150 \mathrm{~mm}$ analytical column, $5 \mu \mathrm{m}$ particle size). The mobile phase consisted of acetonitrile: water $(50: 50, \mathrm{v} / \mathrm{v})$ and the flow rate was $1 \mathrm{~mL} / \mathrm{min}$. The quantity of Ptx was measured by the UV absorbance at the wavelength of $227 \mathrm{~nm}$. EE was calculated as follows:

$$
\begin{aligned}
\mathrm{EE}\left(\%, \frac{\mathrm{w}}{\mathrm{w}}\right) & \\
= & \frac{\text { weight of drug in liposome }}{\text { weight of drug used in preparation of liposome }} \\
& \times 100 .
\end{aligned}
$$

(3) Measurement of Zeta Potential of Liposomes. The zeta potential of prepared liposomes was measured by the ELS8000 zeta potential analyzer among different batches to assess the surface charge and the stability of the liposomes. Zeta potential of liposomes was measured in aqueous dispersion (PBS, pH 7.4). The presented results (mean \pm S.D.) are representatives of independent experiments $(n=5)$.

\section{(4) Morphological Characterization}

(A) Scanning Electron Microscopic (SEM) Observation. The morphology of PEGylated and folate-PEGylated liposomes was observed using field-emission SEM. The PEGylated liposome samples were coated with platinum ( $20 \mathrm{~nm}$ thick) using an ion sputter (JFC-1100, JEOL Ltd., Tokyo, Japan) for 5 minutes at $20 \mathrm{~mA}$. Observation was performed at an accelerating voltage of $5 \mathrm{kV}$ and a working distance of $10 \mathrm{~mm}$. The SEM images were photographed at 10,000x and 50,000x, respectively.

(B) Transmission Electron Microscopic (TEM) Observation. A drop of PEGylated and folate-PEGylated liposomal solution containing $1 \%$ of phosphotungstic acid ( $\mathrm{pH} 7.0$ adjusted with $1 \mathrm{M} \mathrm{KOH}$ solution) was placed on carbon film coated on a copper grid for TEM. Observation was performed at $80 \mathrm{kV}$ in a JEM-2000 FXII. The TEM images were photographed at $50,000 \mathrm{x}$ and $100,000 \mathrm{x}$, respectively.

(5) Stability Test at Storage Condition. The stability of liposomes in $\mathrm{pH}$ 7.4 PBS solution at storage conditions was determined by changes in particle size and EE over a 5-day period during storage at $4^{\circ} \mathrm{C}$. Samples were taken at different time intervals over 24 hours and particle size and EE were obtained immediately. The size of each sample was measured by the particle size analyzer and EE was measured by HPLC assay. Each experiment was repeated at least five times.

(6) In Vitro Release Study. For the in vitro release test of Ptx from PADEXOL Inj., PEGylated liposomes and folatePEGylated liposomes at $\mathrm{pH} 7.4$ phosphate buffer medium was prepared by the dialysis method. Each preparation containing Ptx was diluted with medium and the final concentration of Ptx was adjusted to $90 \mu \mathrm{g} / \mathrm{mL}$. Each sample was put into a dialysis tube (MWCO: 12,000) which was placed into a $50 \mathrm{~mL}$ screw-capped Falcon tube with $10 \mathrm{~mL}$ of dissolution medium. The Falcon tubes were incubated in a shaking water bath thermostated at $37^{\circ} \mathrm{C}$ and shaken at a rate of $100 \mathrm{opm}$. We changed whole media for new media to prevent drug saturation after gathering samples of dissolution media. At 
the predetermined time intervals $(3,6,12,24,48,72,120$, $144,168,192$, and 240 hours after starting incubation), whole medium $(10 \mathrm{~mL})$ was taken and replaced with the same volume of fresh medium $(10 \mathrm{~mL})$. The amount of Ptx released was determined by HPLC.

(7) In Vivo Study. The femoral vein and artery were cannulated with a polyethylene tube (PE-50, Intramedic, Clay Adams Co., Parsippany, NJ, USA) under light ether anesthesia. Cannulated rats were kept in restraining cages under normal housing conditions for 1 2 hours until they recovered from anesthesia prior to the experiments. The rats were divided into three groups (five animals in each group), (1) PADEXOL Inj., commercial i.v. injection of Ptx, (2) PEGylated liposomes containing Ptx for i.v. administration, and (3) folate-PEGylated liposomes containing Ptx for i.v. administration. A single dose (5 mg/kg as Ptx) of each Ptx formulation was given to rats intravenously at the same time [40]. At predetermined time intervals $(0.083,0.25,0.5,0.75,1$, $2,4,6,8$, and 12 hours), whole blood samples (about $350 \mu \mathrm{L}$ ) were withdrawn via the femoral artery into a Vacutainer tube with EDTA. After immediate centrifugation (10,000 g, 10 minutes), the plasma was taken and stored at $4^{\circ} \mathrm{C}$ until it was assayed within 24 hours. Whole blood (approximately $1 \mathrm{~mL}$ ) collected from untreated rats was infused via the femoral artery at $0.5,2$, and 8 hours to replace blood-loss due to blood sampling. Also, in order to evaluate the lymphatic delivery of Ptx, the rats were also divided into three groups (five animals in each group) and administered each formulation as mentioned above. At 4 hours after administration, whole blood samples were taken via the abdominal aorta and centrifuged immediately (10,000 g, 10 minutes), and lymph nodes such as mesenteric and axillary lymph node were isolated and weighed ( $25 \mathrm{mg}$ for each lymph node). These lymph node samples were suspended by a homogenizer for 1 minute in PBS ( $\mathrm{pH} 7.4$ ) to achieve a final concentration of $25 \mathrm{mg} / \mathrm{mL}$ in the homogenate and stored at $-80^{\circ} \mathrm{C}$ until being assayed.

(8) Determination of Ptx in Rat Plasma and Lymph Node Suspension. The plasma concentrations of Ptx were determined by HPLC assay and modification of the method reported previously [41-43]. The HPLC conditions for the determination of Ptx in rat plasma and lymph node suspension are the same as mentioned above ((2) determination of drug encapsulation efficiency). The stock solutions of Ptx $(1 \mathrm{mg} / \mathrm{mL})$ were prepared in methanol and stored at $4^{\circ} \mathrm{C}$. The stock solution of glimepiride $(100 \mu \mathrm{g} / \mathrm{mL})$ for internal standard (I.S.) was prepared by dissolving glimepiride in ethanol. The calibration curves were obtained by plotting the peak-area ratios of the drug to I.S. versus the concentration of Ptx. Calibration standards at rat plasma and lymph node suspension of Ptx were prepared at concentrations of 10, 20, $50,100,200,500,1000$, and $10000 \mathrm{ng} / \mathrm{mL}$ in drug-free pooled rat plasma and lymph node suspension. Briefly, $100 \mu \mathrm{L}$ of rat plasma or lymph node suspension sample was put into a test tube and $10 \mu \mathrm{L}$ of I.S. (glimepiride in ethanol diluted with deionized water at a concentration of $5 \mu \mathrm{g} / \mathrm{mL}$ ) was added. After vortexing for 30 seconds, $2 \mathrm{~mL}$ of dichloromethane was added and vortexed for 2 minutes. The extract was then centrifuged for 10 minutes at $10,000 \mathrm{~g}\left(4^{\circ} \mathrm{C}\right)$. After aspirating the upper layer, $1.5 \mathrm{~mL}$ of the lower layer was transferred to the new test tube for centrifugal evaporation for 1.5 hours at $20,000 \mathrm{~g}$ at $45^{\circ} \mathrm{C}$ under nitrogen stream. The residue was reconstituted with $100 \mu \mathrm{L}$ of mobile phase by vortexing for 30 seconds and $50 \mu \mathrm{L}$ of the supernatant was injected into the HPLC system. The intra- and inter-day assay precision as well as the accuracy fulfilled the FDA guidelines [44], and the mean absolute recoveries from plasma and lymph node were $95.4 \pm 3.7$ and $96.8 \pm 4.7 \%$, respectively. These results support the idea that this method was applicable to the kinetics of Ptx for plasma and lymph node suspension.

(9) Pharmacokinetic Analysis and Lymphatic Delivery Evaluation. The pharmacokinetic parameters associated with i.v. administration were estimated by noncompartmental methods using WinNonlin program [45]. The Ptx pharmacokinetic parameters, such as $\mathrm{CL}_{t}$ and $t_{1 / 2}$, were calculated from standard equations [46]. $C_{\max }$ was directly obtained by observation. The targeting efficiency of Ptx to the lymphatic system was calculated as the ratio of Ptx concentration in lymph nodes (mesenteric and axillary nodes) to the concentration in rat plasma at 4 hours after i.v. administration of each formulation.

2.2.4. Statistical Evaluation. All calculated values were expressed as their mean \pm S.D. All data were analyzed for statistical significance by Student's $t$-test with $P<0.05$ indicating a significant difference.

\section{Results and Discussion}

\subsection{Preparation of PEGylated and Folate-PEGylated Liposomes}

3.1.1. Determination of Optimal Concentration of DSPE$m P E G$. EPC:CHOL:Ptx mixture was used with $20: 10: 1$ molar ratio. The concentrations of DSPE-mPEG were varied from 0 to 4 molar ratio with respect to the amount of modifier in the liposome composition. When encapsulated in the appropriated concentrations of DSPE-mPEG, DSPEmPEG has been shown to increase encapsulation efficacy [47] and to reduce the leakage of encapsulated drugs [48]. Our results showed that the encapsulation efficiency was found to increase between 0 and 3 molar ratio of DSPE-mPEG. But little change was observed at 4 molar ratio. Also, liposomal size was decreased with increasing DSPE-mPEG molar ratio, but there is not a remarkable difference between 3 and 4 molar ratio. The liposomal size was $114 \pm 6.81 \mathrm{~nm}$ and EE was $81 \pm 2.3 \%$. From these results, the optimal concentration was determined at 3 molar ratio of DSPE-mPEG (Table 1).

3.1.2. Determination of Optimal Concentration of Folate$P E G-D S P E$. We also determined the optimal molar ratio of folate-PEG-DSPE. EPC : CHOL : Ptx mixture $(20: 10: 1$ molar ratio) was used. The concentrations of folate-PEG-DSPE (DSPE-mPEG : folate-PEG-DSPE $=9: 1$ ) varied from 0 to 4 molar ratio with respect to the amount of modifier in the liposome composition. Our results showed that the encapsulation efficiency increased between 0 and 3 molar ratio of 
TABLE 1: Change of liposomal size and drug EE of PEGylated and folate-PEGylated liposomes containing Ptx various molar ratios of DSPE$\mathrm{mPEG}^{\#}$.

\begin{tabular}{lcccc}
\hline \multirow{2}{*}{ Molar ratio of DSPE-mPEG } & \multicolumn{2}{c}{ Liposomal size $(\mathrm{nm})$} & \multicolumn{2}{c}{ Encapsulation efficiency (\%) } \\
& PEGylated liposome & Folate-PEGylated liposome & PEGylated liposome & Folate-PEGylated liposome \\
\hline 0 & $224 \pm 21.8$ & $224 \pm 21.8$ & $76 \pm 1.8$ & $76 \pm 1.8$ \\
1 & $148 \pm 3.65^{*}$ & $140 \pm 9.79^{*}$ & $78 \pm 3.6$ & $77 \pm 1.3$ \\
2 & $123 \pm 10.2^{*}$ & $126 \pm 6.65^{*}$ & $79 \pm 2.7$ & $82 \pm 1.2^{*}$ \\
3 & $114 \pm 6.81^{*}$ & $122 \pm 4.87^{*}$ & $81 \pm 2.3^{*}$ & $88 \pm 2.0^{*}$ \\
4 & $121 \pm 20.0^{*}$ & $125 \pm 3.40^{*}$ & $83 \pm 4.8^{*}$ & $68 \pm 1.3^{*}$ \\
\hline
\end{tabular}

${ }^{\#}$ Mean \pm S.D. $(n=5) .{ }^{*} P<0.05$ between 0 and specific molar ratio of DSPE-mPEG.

folate-PEG-DSPE, but little change was observed at 4 molar ratio. Also, liposomal size decreased with increasing folatePEG-DSPE molar ratio, but there is not a remarkable difference between 3 and 4 molar ratio. The liposomal size was $122 \pm 4.87 \mathrm{~nm}$ and EE was $88 \pm 2.0 \%$. We conclude that liposomal size slightly increased (from 114 to $122 \mathrm{~nm}$ ). Encapsulation efficiency was also increased (from 81 to $88 \%$ ) due to the addition of folate to PEG-DSPE liposomes. From these results, the optimal concentration was at 3 molar ratio of folate-PEG-DSPE (Table 1).

\subsubsection{Zeta Potential of PEGylated and Folate-PEGylated Lipo-} somes. The zeta potential value is an important characteristic, and it can influence liposome stability and mucoadhesion. Our results of zeta potential were $-27.7 \pm 5.51$ and $-42.9 \pm$ $6.28 \mathrm{mV}$ for PEGylated and folate-PEGylated liposomes, respectively. Therefore, we expected that prepared liposomes can increase the residence time of anticancer drugs in the circulation, enhance drug accumulation in tumors, and be well-distributed to other tissues [49].

3.2. Morphological Characterization of PEGylated and FolatePEGylated Liposomes. In the SEM observation (Figure 1), the PEGylated and folate-PEGylated liposomes containing Ptx prepared from above optimal conditions were about $100 \mathrm{~nm}$ in size and had a comparatively spherical shape with a smooth surface, which were almost the same results in the TEM observation (Figure 2) as well as DLS analyzer measurements. These results have shown that PEGylated and folate-PEGylated liposomes containing Ptx having a particle size of about $100 \mathrm{~nm}$ and a nice morphology were successfully prepared by this method.

3.3. Stability Test of Liposomes at Storage Condition. As shown in Table 2, the particle size and EE of both PEGylated and folate-PEGylated liposomes in $\mathrm{pH}$ 7.4 PBS solution were insignificantly $(P>0.05)$ changed during 5 days. But EE had tendency to be slightly decreased as time went by. We considered that this result was due to the release of Ptx which was attached to the surface of the liposomes. We think that the liposomal formulations showed excellent colloidal stability and drug retention during this period. And there were no visible changes to the physical appearance of the formulation or signs of drug precipitation. The results showed that the prepared liposomes in $\mathrm{pH}$ 7.4 PBS solution were stable at least for 5 days at storage conditions $\left(4^{\circ} \mathrm{C}\right)$.
3.4. In Vitro Release Study. Figure 3 compares the release profiles of Ptx from PADEXOL Inj., PEGylated liposomes, and folate-PEGylated liposomes in $\mathrm{pH} 7.4$ phosphate buffer medium. The release profiles between PEGylated and folatePEGylated liposomes containing Ptx were similar and showed significantly lower profiles than PADEXOL Inj. $(P<$ $0.05)$. The average release ratio of folate-PEGylated liposomes tended to be relatively small compared to that of PEGylated liposomes. This result is likely due to the addition of folate to PEGylated liposomes. Therefore, the PEGylated and folatePEGylated liposomes containing Ptx have a potential application for controlled release into the blood and lymphatic system.

\subsection{Pharmacokinetic Analysis and Lymphatic Targeting Effi-} ciency Evaluation. Figure 4 shows the mean concentrationtime profiles of Ptx in rat plasma after i.v. administration of PADEXOL Inj., PEGylated liposome containing Ptx, and folate-PEGylated liposomes containing Ptx ( $5 \mathrm{mg} / \mathrm{kg}$ as Ptx) $(n=5)$. Table 3 lists the pharmacokinetic parameters obtained by the noncompartmental method using WinNonlin. After i.v. administration, $t_{1 / 2}, \mathrm{CL}_{t}$, and $C_{\max }$ of PEGylated and folate-PEGylated liposomes containing Ptx were significantly different $(P<0.05)$ from those of PADEXOL Inj. The $t_{1 / 2}$ of the prepared liposomes was about three times higher than that of PADEXOL Inj. and the $\mathrm{CL}_{t}$ was about three times lower than that of PADEXOL Inj. These results showed that the prepared liposomes circulated in the blood for a longer period of time and were consistent with the expectation from the in vitro release study. The pharmacokinetic parameters of Ptx reported that the $t_{1 / 2}$ was 2.3 hours and the $\mathrm{CL}_{t}$ was $775 \mathrm{~mL} / \mathrm{hr} \cdot \mathrm{kg}$ at the i.v. dose of $5 \mathrm{mg} / \mathrm{kg}$ [50]. In a previously related study, the $\mathrm{CL}_{t}$ of paclitaxel solution at the i.v. dose of $7.5 \mathrm{mg} / \mathrm{kg}$ was $1677.85 \mathrm{~mL} / \mathrm{hr} \cdot \mathrm{kg}$ [51]. Based on these results, we conclude that the $\mathrm{CL}_{t}$ in our study is comparable with that in related studies. The concentrations of Ptx in mesenteric and axillary lymph nodes after i.v. administration were shown in Table 3. There was a significant difference between the prepared liposomes containing Ptx and control formulation (PADEXOL Inj.) $(P<0.05)$. In the case of PEGylated and folate-PEGylated liposomes, the concentration of Ptx in the lymph nodes was lower compared to the control formulation. The observed difference in these data could be related to the difference in their biocompatibility. A large number of lymphocytes, such as macrophages or leukocytes, are known to be distributed in the lymph nodes and lymphatic capillaries. 


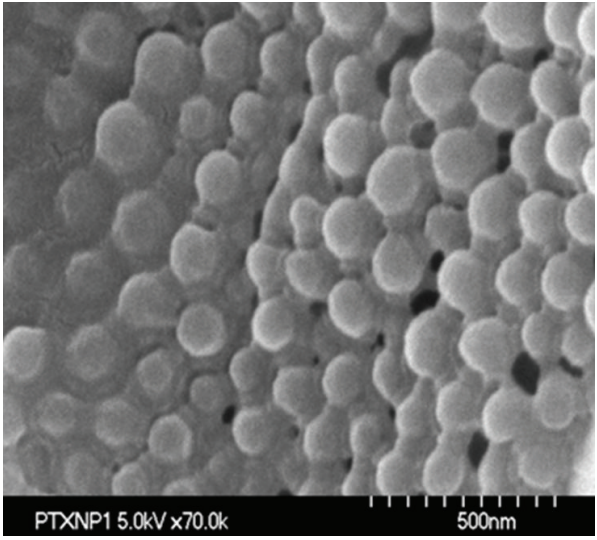

(a)

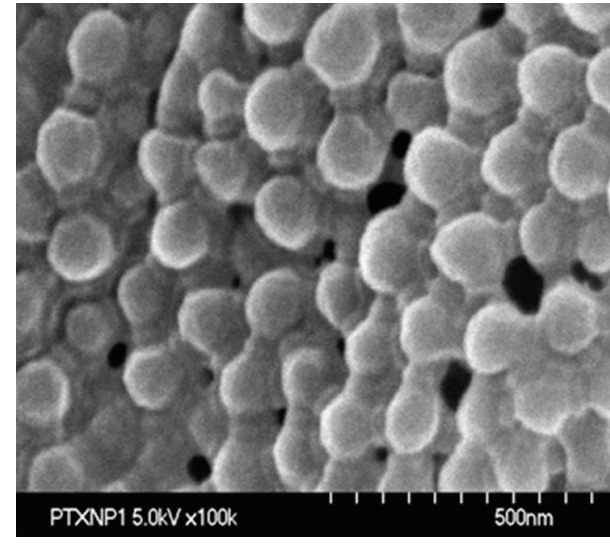

(b)

Figure 1: SEM image of (a) PEGylated liposomes containing Ptx (70,000x) and (b) folate-PEGylated liposomes containing Ptx (100,000x).

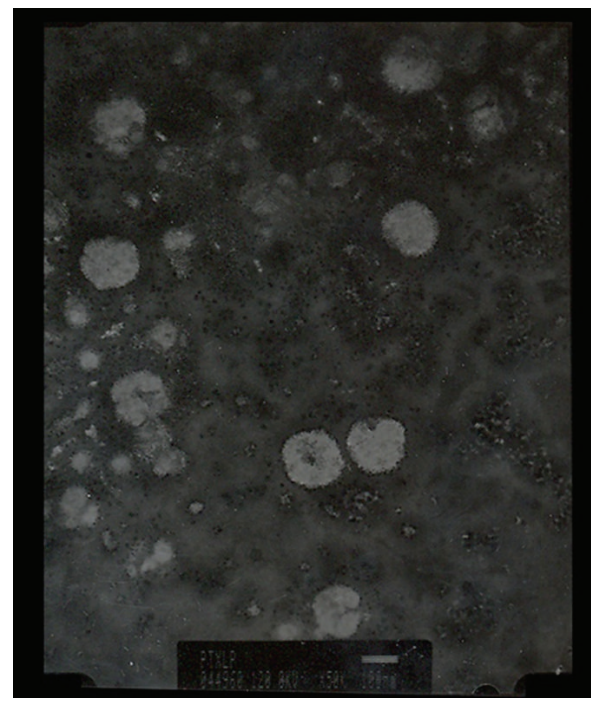

(a)

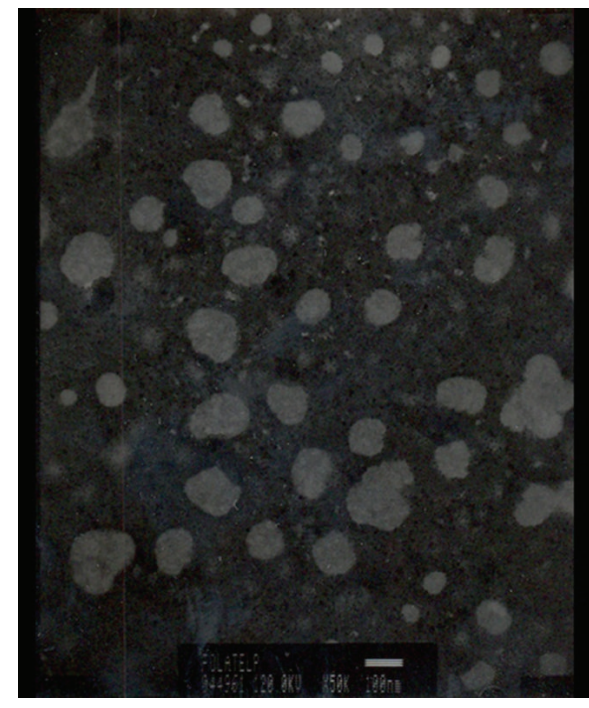

(b)

FIgure 2: TEM image of (a) PEGylated liposomes containing Ptx (bar = $100 \mathrm{~nm})$ and $(\mathrm{b})$ folate-PEGylated liposomes containing Ptx $($ bar $=$ $100 \mathrm{~nm}$ ).

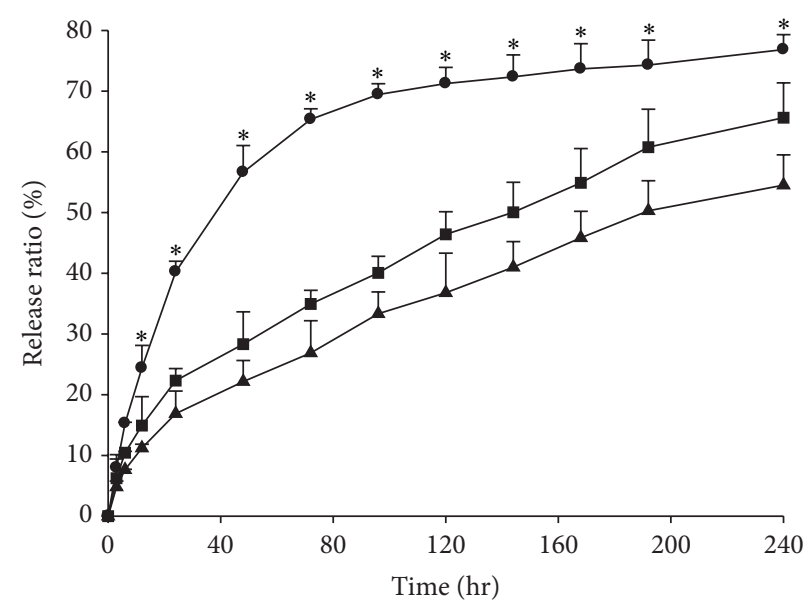

Figure 3: In vitro release profiles of Ptx from PADEXOL Inj. (•), PEGylated liposomes ( $\bullet$ ), and folate-PEGylated liposomes (ム) in dissolution media (pH 7.4). Each value represents the mean \pm S.D. $(n=5) .{ }^{*} P<0.05$ between PADEXOL Inj. and the prepared liposomes. 
TABLE 2: The changes of liposomal size and encapsulation efficiency of PEGylated and folate-PEGylated liposomes at $4^{\circ} \mathrm{C}$ for 120 hours ${ }^{\#}$.

\begin{tabular}{lcccc}
\hline \multirow{2}{*}{ Time (hour) } & \multicolumn{2}{c}{ Liposomal size (nm) } & \multicolumn{2}{c}{ Encapsulation efficiency (\%) } \\
& PEGylated liposome & Folate-PEGylated liposome & PEGylated liposome & Folate-PEGylated liposome \\
\hline 0 & $114 \pm 6.81$ & $122 \pm 4.87$ & $81 \pm 2.3$ & $88 \pm 2.0$ \\
24 & $115 \pm 4.26$ & $128 \pm 5.02$ & $81 \pm 2.0$ & $86 \pm 1.3$ \\
48 & $115 \pm 2.57$ & $128 \pm 4.38$ & $80 \pm 3.5$ & $88 \pm 1.4$ \\
72 & $114 \pm 3.91$ & $129 \pm 4.26$ & $80 \pm 3.1$ & $86 \pm 1.8$ \\
96 & $115 \pm 4.44$ & $129 \pm 4.24$ & $79 \pm 3.0$ & $86 \pm 1.1$ \\
120 & $115 \pm 2.71$ & $128 \pm 6.84$ & $79 \pm 1.8$ & $85 \pm 2.5$ \\
\hline
\end{tabular}

${ }^{\#}$ Mean \pm S.D. $(n=5)$.

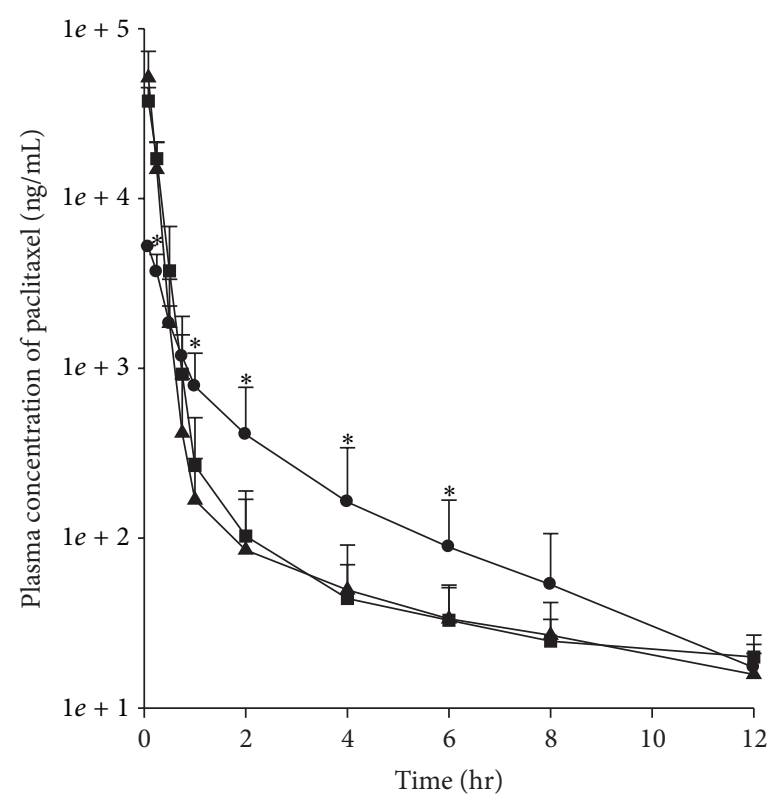

FIgURE 4: Mean plasma concentration-time curves of Ptx after i.v. administration (5 mg/kg) of PADEXOL Inj. (•), PEGylated liposomes containing Ptx ( $\bullet)$, and folate-PEGylated liposomes containing Ptx $(\boldsymbol{\Delta})$ to rats. Each value represents the mean \pm S.D. $(n=5)$. ${ }^{*} P<0.05$ between PADEXOL Inj. and the prepared liposomes.

Thus, although the blood concentrations of Ptx in the prepared liposomes at 4 hours after i.v. administration $(5 \mathrm{mg} / \mathrm{kg})$ to rats were significant $(P<0.05)$ lower than those in the control formulation (PADEXOL Inj.), on the contrary, the relative delivered effects of Ptx in the prepared liposomes on both lymph nodes compared to the control formulation increased due to their biocompatibility, phagocytosis by enriched macrophages, and so on.

Lymphatic targeting efficiency of Ptx into the mesenteric and axillary lymph nodes was shown in Table 3. After i.v. administration, the lymphatic targeting efficiency of PEGylated and folate-PEGylated liposomes containing Ptx was significantly increased when compared with that of PADEXOL Inj. $(P<0.05)$ in both lymph nodes. This increased lymphatic targeting efficiency can lower the drug concentration needed to have an effect. Delivering with the prepared liposomes increased not only the time of the drug circulation but also it seems to increase the efficacy at the target sites and reduce the systemic side effects as we expected. These results suggest that PEGylated and folate-PEGylated liposomes containing
Ptx could be as effective for targeting delivery of Ptx to the lymphatic system as the i.v. formulation.

\section{Conclusions}

The PEGylated and folate-PEGylated liposomes containing Ptx with diameters of about $120 \mathrm{~nm}$ could be prepared easily and are reproducible by lipid film hydration method. The drug EE was above $80 \%$ at the optimal molar ratio. From in vivo studies, the $t_{1 / 2}$ and $\mathrm{CL}_{t}$ of the prepared liposomes were significantly different from those of PADEXOL Inj. $(P<$ 0.05). The prepared liposomes showed significantly higher lymphatic targeting efficiency than that of PADEXOL Inj. at the mesenteric and axillary lymph nodes $(P<0.05)$. Generally, most of the nanosized prepared liposomes accumulate to the target lymph node during continuous systemic circulation due to the physicochemical characteristics such as particle size and biodegradable polymers coating the surface. The prepared liposomes can easily pass through the walls of lymphatic capillaries during systemic circulation, and this 
TABLE 3: Pharmacokinetic parameters and targeting efficiencies of Ptx to mesenteric and axillary lymph nodes at 4 hours after i.v. administration $(5 \mathrm{mg} / \mathrm{kg})$ of the prepared PEGylated and folate-PEGylated liposomes containing Ptx and PADEXOL Inj. to rats" .

\begin{tabular}{|c|c|c|c|}
\hline Parameters & PADEXOL Inj. & PEGylated liposome & Folate-PEGylated liposome \\
\hline$t_{1 / 2}$ (hour) & $3.43 \pm 1.35$ & $10.8 \pm 3.51^{*}$ & $9.14 \pm 2.26^{*}$ \\
\hline $\mathrm{CL}_{t}(\mathrm{~mL} / \mathrm{hr} \cdot \mathrm{kg})$ & $1285 \pm 317.0$ & $413.5 \pm 106.1^{*}$ & $380.3 \pm 115.7^{*}$ \\
\hline$C_{\max }(\mu \mathrm{g} / \mathrm{mL})$ & $5.16 \pm 1.30$ & $37.5 \pm 7.47^{*}$ & $51.4 \pm 12.3^{*}$ \\
\hline \multicolumn{4}{|c|}{ Concentration (ng/g lymph node) } \\
\hline Mesenteric lymph node & $1364 \pm 102.2$ & $740.0 \pm 236.1^{*}$ & $810.4 \pm 247.0^{*}$ \\
\hline Axillary lymph node & $1304 \pm 112.7$ & $672.8 \pm 182.1^{*}$ & $712.3 \pm 102.4^{*}$ \\
\hline \multicolumn{4}{|l|}{ Targeting efficiency } \\
\hline Mesenteric lymph node & $8.06 \pm 1.04$ & $15.1 \pm 4.14^{*}$ & $18.8 \pm 4.81^{*}$ \\
\hline Axillary lymph node & $8.01 \pm 1.11$ & $13.9 \pm 2.98^{*}$ & $16.6 \pm 3.18^{*}$ \\
\hline
\end{tabular}

${ }^{\#}$ Mean \pm S.D. $(n=5) .{ }^{*} P<0.05$ between PADEXOL Inj. and the prepared liposomes.

passive targeting enables the drug to be targeted to the lymphatic system. Based on the results of our study, we conclude that this mechanism is related to the drug delivery system of the prepared liposomes. Therefore, it was suggested that the prepared liposomes have an effect on targeting Ptx to the lymph node, and the prepared liposomes are good candidates for delivering Ptx to the lymphatic system.

\section{Conflict of Interests}

The authors declare that there is no conflict of interests regarding the publication of this paper.

\section{Authors' Contribution}

Hea-Young Cho and Chong Ki Lee contributed equally to this work.

\section{Acknowledgment}

This work was supported by the National Research Foundation of Korea (NRF) Grant funded by the Ministry of Education, Science and Technology (MEST) (no. 2011-0029209).

\section{References}

[1] M. E. Wall and M. C. Wani, "Camptothecin and taxol: from discovery to clinic," Journal of Ethnopharmacology, vol. 51, no. 1-3, pp. 239-254, 1996.

[2] J. G. Kuhn, "Pharmacology and pharmacokinetics of paclitaxel," Annals of Pharmacotherapy, vol. 28, no. 5, pp. S15-S17, 1994.

[3] F. A. Fitzpatrick and R. Wheeler, "The immunopharmacology of paclitaxel (Taxol), docetaxel (Taxotere), and related agents," International Immunopharmacology, vol. 3, no. 13-14, pp. 16991714, 2003.

[4] K. A. Kurdziel, D. O. Kiesewetter, R. E. Carson, W. C. Eckelman, and P. Herscovitch, "Biodistribution, radiation dose estimates, and in vivo Pgp modulation studies of ${ }^{18} \mathrm{~F}$-paclitaxel in nonhuman primates," Journal of Nuclear Medicine, vol. 44, no. 8, pp. 1330-1339, 2003.

[5] L.-Q. Yang, B. Wang, H. Gan et al., "Enhanced oral bioavailability and anti-tumour effect of paclitaxel by 20(s)-ginsenoside Rg3 in vivo," Biopharmaceutics and Drug Disposition, vol. 33 , no. 8 , pp. 425-436, 2012.

[6] P. Engblom, J. O. Pulkkinen, V. Rantanen et al., "Effects of paclitaxel with or without cremophor EL on cellular clonogenic survival and apoptosis," European Journal of Cancer, vol. 35, no. 2, pp. 284-288, 1999.

[7] J. Szebeni, F. M. Muggia, and C. R. Alving, "Complement activation by Cremophor EL as a possible contributor to hypersensitivity to paclitaxel: an in vitro study," Journal of the National Cancer Institute, vol. 90, no. 4, pp. 300-306, 1998.

[8] H. Gelderblom, J. Verweij, K. Nooter, and A. Sparreboom, "Cremophor EL: the drawbacks and advantages of vehicle selection for drug formulation," European Journal of Cancer, vol. 37, no. 13, pp. 1590-1598, 2001.

[9] G. J. Fetterly and R. M. Straubinger, "Pharmacokinetics of paclitaxel-containing liposomes in rats," AAPS PharmSciTech, vol. 5, no. 5, pp. 90-100, 2003.

[10] T. Yang, F.-D. Cui, M.-K. Choi et al., "Enhanced solubility and stability of PEGylated liposomal paclitaxel: in vitro and in vivo evaluation," International Journal of Pharmaceutics, vol. 338, no. 1-2, pp. 317-326, 2007.

[11] J.-S. Choi and S.-C. Shin, "Enhanced paclitaxel bioavailability after oral coadministration of paclitaxel prodrug with naringin to rats," International Journal of Pharmaceutics, vol. 292, no. 1-2, pp. 149-156, 2005.

[12] S. M. Azouz, J. Walpole, S. Amirifeli, K. N. Taylor, M. W. Grinstaff, and Y. L. Colson, "Prevention of local tumor growth with paclitaxel-loaded microspheres," Journal of Thoracic and Cardiovascular Surgery, vol. 135, no. 5, pp. 1014-1021, 2008.

[13] K. Guo and C. C. Chu, "Biodegradable and injectable paclitaxelloaded poly(ester amide)s microspheres: fabrication and characterization," Journal of Biomedical Materials Research Part B Applied Biomaterials, vol. 89, no. 2, pp. 491-500, 2009.

[14] H. Suh, B. Jeong, R. Rathi, and S. W. Kim, "Regulation of smooth muscle cell proliferation using paclitaxel-loaded poly (ethylene oxide)-poly (lactide/glycolide) nanospheres," Journal of Biomedical Materials Research Part A, vol. 42, no. 2, pp. 331338, 1998.

[15] S.-S. Feng and G. Huang, "Effects of emulsifiers on the controlled release of paclitaxel (Taxol) from nanospheres of biodegradable polymers," Journal of Controlled Release, vol. 71, no. 1, pp. 53-69, 2001.

[16] S. Yang, R. N. Gursoy, G. Lambert, and S. Benita, "Enhanced oral absorption of paclitaxel in a novel self-microemulsifying 
drug delivery system with or without concomitant use of Pglycoprotein inhibitors," Pharmaceutical Research, vol. 21, no. 2, pp. 261-270, 2004.

[17] P. Zhao, H. Wang, M. Yu et al., "Paclitaxel loaded folic acid targeted nanoparticles of mixed lipid-shell and polymer-core: In vitro and in vivo evaluation," European Journal of Pharmaceutics and Biopharmaceutics, vol. 81, no. 2, pp. 248-256, 2012.

[18] V. Zabaleta, G. Ponchel, H. Salman, M. Agüeros, C. Vauthier, and J. M. Irache, "Oral administration of paclitaxel with pegylated poly(anhydride) nanoparticles: permeability and pharmacokinetic study," European Journal of Pharmaceutics and Biopharmaceutics, vol. 81, no. 3, pp. 514-523, 2012.

[19] S. J. Torne, K. A. Ansari, P. R. Vavia, F. Trotta, and R. Cavalli, "Enhanced oral paclitaxel bioavailability after administration of paclitaxel-loaded nanosponges," Drug Delivery, vol. 17, no. 6, pp. 419-425, 2010.

[20] B. Mognetti, A. Barberis, S. Marino et al., "In vitro enhancement of anticancer activity of paclitaxel by a Cremophor free cyclodextrin-based nanosponge formulation," Journal of Inclusion Phenomena and Macrocyclic Chemistry, vol. 74, no. 1-4, pp. 201-210, 2012.

[21] M. V. S. Varma and R. Panchagnula, "Enhanced oral paclitaxel absorption with vitamin E-TPGS: effect on solubility and permeability in vitro, in situ and in vivo," European Journal of Pharmaceutical Sciences, vol. 25, no. 4-5, pp. 445-453, 2005.

[22] J. D. Kingsley, H. Dou, J. Morehead, B. Rabinow, H. E. Gendelman, and C. J. Destache, "Nanotechnology: a focus on nanoparticles as a drug delivery system," Journal of Neuroimmune Pharmacology, vol. 1, no. 3, pp. 340-350, 2006.

[23] C. X. Song, V. Labhasetwar, H. Murphy et al., "Formulation and characterization of biodegradable nanoparticles for intravascular local drug delivery," Journal of Controlled Release, vol. 43, no. 2, pp. 197-212, 1997.

[24] I. Brigger, C. Dubernet, and P. Couvreur, "Nanoparticles in cancer therapy and diagnosis," Advanced Drug Delivery Reviews, vol. 54, no. 5, pp. 631-651, 2002.

[25] R. H. Muller and C. M. Keck, "Challenges and solutions for the delivery of biotech drugs: a review of drug nanocrystal technology and lipid nanoparticles," Journal of Biotechnology, vol. 113, no. 1-3, pp. 151-170, 2004.

[26] G. Blume and G. Cevc, "Liposomes for the sustained drug release in vivo," Biochimica et Biophysica Acta-Biomembranes, vol. 1029, no. 1, pp. 91-97, 1990.

[27] A. L. Klibanov, K. Maruyama, V. P. Torchilin, and L. Huang, "Amphipathic polyethyleneglycols effectively prolong the circulation time of liposomes," FEBS Letters, vol. 268, no. 1, pp. 235237, 1990.

[28] S. D. Weitman, R. H. Lark, L. R. Coney et al., "Distribution of the folate receptor GP38 in normal and malignant cell lines and tissues," Cancer Research, vol. 52, no. 12, pp. 3396-3401, 1992.

[29] G. Toffoli, C. Cernigoi, A. Russo, A. Gallo, M. Bagnoli, and M. Boiocchi, "Overexpression of folate binding protein in ovarian cancers," International Journal of Cancer, vol. 74, no. 2, pp. 193198, 1997.

[30] A. Gabizon, H. Shmeeda, A. T. Horowitz, and S. Zalipsky, "Tumor cell targeting of liposome-entrapped drugs with phospholipid-anchored folic acid-PEG conjugates," Advanced Drug Delivery Reviews, vol. 56, no. 8, pp. 1177-1192, 2004.

[31] A. R. Hilgenbrink and P. S. Low, "Folate receptor-mediated drug targeting: from therapeutics to diagnostics," Journal of Pharmaceutical Sciences, vol. 94, no. 10, pp. 2135-2146, 2005.
[32] J. M. Saul, A. Annapragada, J. V. Natarajan, and R. V. Bellamkonda, "Controlled targeting of liposomal doxorubicin via the folate receptor in vitro," Journal of Controlled Release, vol. 92, no. 1-2, pp. 49-67, 2003.

[33] A. Gabizon, A. T. Horowitz, D. Goren, D. Tzemach, H. Shmeeda, and S. Zalipsky, "In vivo fate of folate-targeted polyethylene-glycol liposomes in tumor-bearing mice," Clinical Cancer Research, vol. 9, no. 17, pp. 6551-6559, 2003.

[34] P. J. Stevens, M. Sekido, and R. J. Lee, "A folate receptor-targeted lipid nanoparticle formulation for a lipophilic paclitaxel prodrug," Pharmaceutical Research, vol. 21, no. 12, pp. 2153-2157, 2004.

[35] H. S. Yoo and T. G. Park, "Folate receptor targeted biodegradable polymeric doxorubicin micelles," Journal of Controlled Release, vol. 96, no. 2, pp. 273-283, 2004.

[36] J. Wu, Q. Liu, and R. J. Lee, "A folate receptor-targeted liposomal formulation for paclitaxel," International Journal of Pharmaceutics, vol. 316, no. 1-2, pp. 148-153, 2006.

[37] R. J. Lee and P. S. Low, "Folate-mediated tumor cell targeting of liposome-entrapped doxorubicin in vitro," Biochimica et Biophysica Acta (BBA)-Biomembranes, vol. 1233, no. 2, pp. 134144, 1995.

[38] H. Takeuchi, H. Yamamoto, T. Niwa, T. Hino, and Y. Kawashima, "Enteral absorption of insulin in rats from mucoadhesive chitosan-coated liposomes," Pharmaceutical Research, vol. 13, no. 6, pp. 896-901, 1996.

[39] T. Yoshida, N. Oide, T. Sakamoto et al., "Induction of cancer cell-specific apoptosis by folate-labeled cationic liposomes," Journal of Controlled Release, vol. 111, no. 3, pp. 325-332, 2006.

[40] S. S. Shord and J. R. Camp, "Intravenous administration of paclitaxel in Sprague-Dawley rats: what is a safe dose?" Biopharmaceutics and Drug Disposition, vol. 27, no. 4, pp. 191-196, 2006.

[41] F. Coudoré, N. Authier, D. Guillaume, A. Béal, E. Duroux, and J. Fialip, "High-performance liquid chromatographic determination of paclitaxel in rat serum: application to a toxicokinetic study," Journal of Chromatography B: Biomedical Sciences and Applications, vol. 721, no. 2, pp. 317-320, 1999.

[42] F. Mohamed, P. Marchettini, O. A. Stuart, and P. H. Sugarbaker, "Pharmacokinetics and tissue distribution of intraperitoneal paclitaxel with different carrier solutions," Cancer Chemotherapy and Pharmacology, vol. 52, no. 5, pp. 405-410, 2003.

[43] J.-S. Choi and X. Li, "The effect of verapamil on the pharmacokinetics of paclitaxel in rats," European Journal of Pharmaceutical Sciences, vol. 24, no. 1, pp. 95-100, 2005.

[44] "Guidance for industry, Bioanalytical method validation," U.S. Food, Drug Administration, 2001, http://www.fda.gov/ downloads/Drugs/GuidanceComplianceRegulatoryInformation/ Guidances/UCM070107.pdf.

[45] WinNonlin, User's Guide Ver. 3.0, Pharsight Corp., Mountain View, Calif, USA, 1999.

[46] M. Gibaldi and D. Perrier, Pharmacokinetics, Marcel Dekker, New York, NY, USA, 2nd edition, 1982.

[47] K. Moribe, K. Maruyama, and M. Iwatsuru, "Encapsulation characteristics of nystatin in liposomes: effects of cholesterol and polyethylene glycol derivatives," International Journal of Pharmaceutics, vol. 188, no. 2, pp. 193-202, 1999.

[48] M. Silvander, M. Johnsson, and K. Edwards, "Effects of PEGlipids on permeability of phosphatidylcholine/cholesterol liposomes in buffer and in human serum," Chemistry and Physics of Lipids, vol. 97, no. 1, pp. 15-26, 1998. 
[49] J.-Y. Fang, "Nano- or submicron-sized liposomes as carriers for drug delivery," Chang Gung Medical Journal, vol. 29, no. 4, pp. 358-362, 2006.

[50] H. Chen, Z. Zhang, C. McNulty et al., "A high-throughput combinatorial approach for the discovery of a Cremophor EL-free paclitaxel formulation," Pharmaceutical Research, vol. 20, no. 8, pp. 1302-1308, 2003.

[51] T. Yang, M.-K. Choi, F.-D. Cui et al., "Preparation and evaluation of paclitaxel-loaded PEGylated immunoliposome," Journal of Controlled Release, vol. 120, no. 3, pp. 169-177, 2007. 

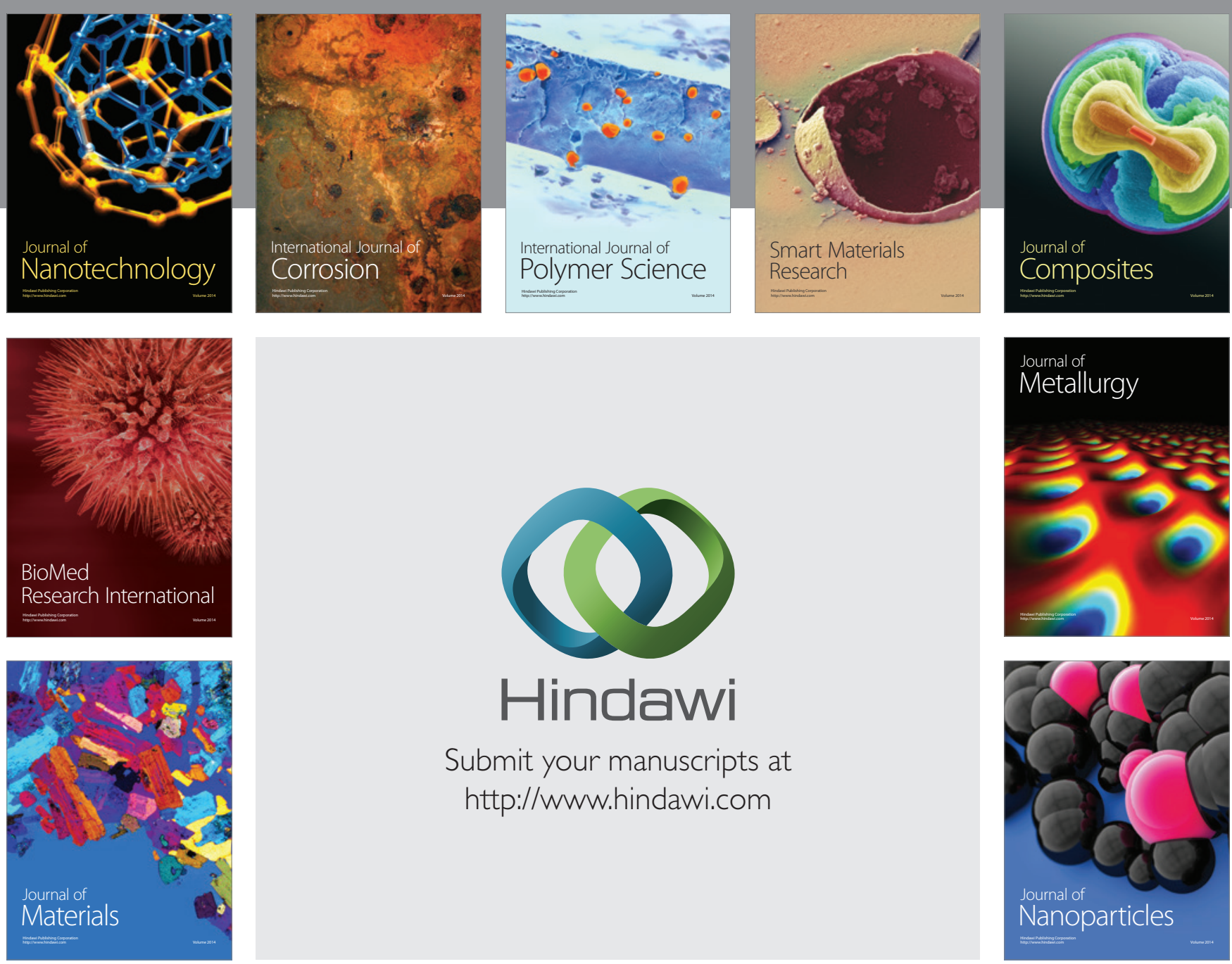

Submit your manuscripts at http://www.hindawi.com
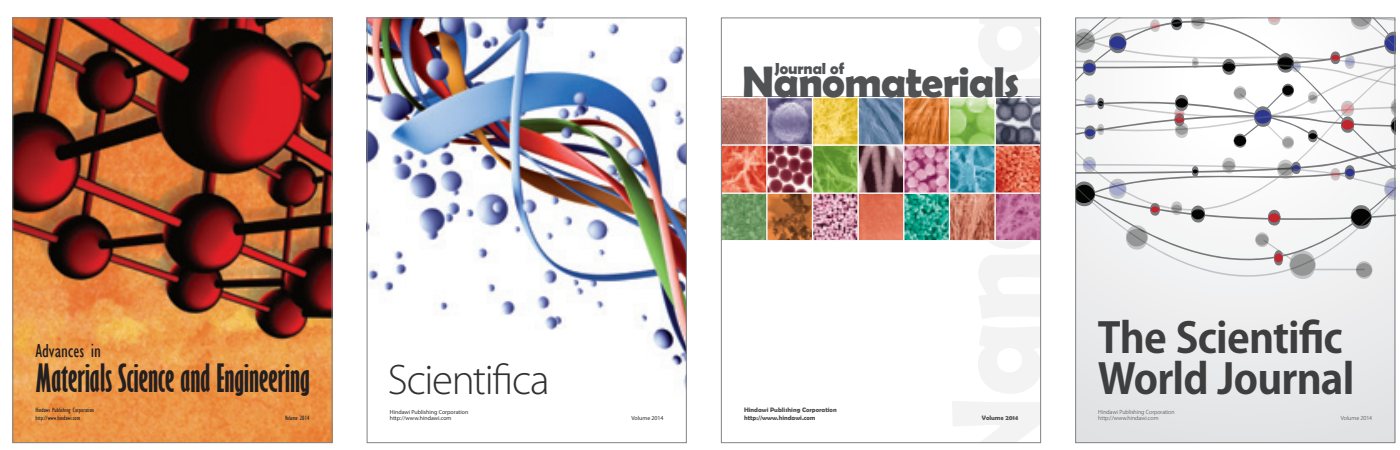

\section{The Scientific World Journal}
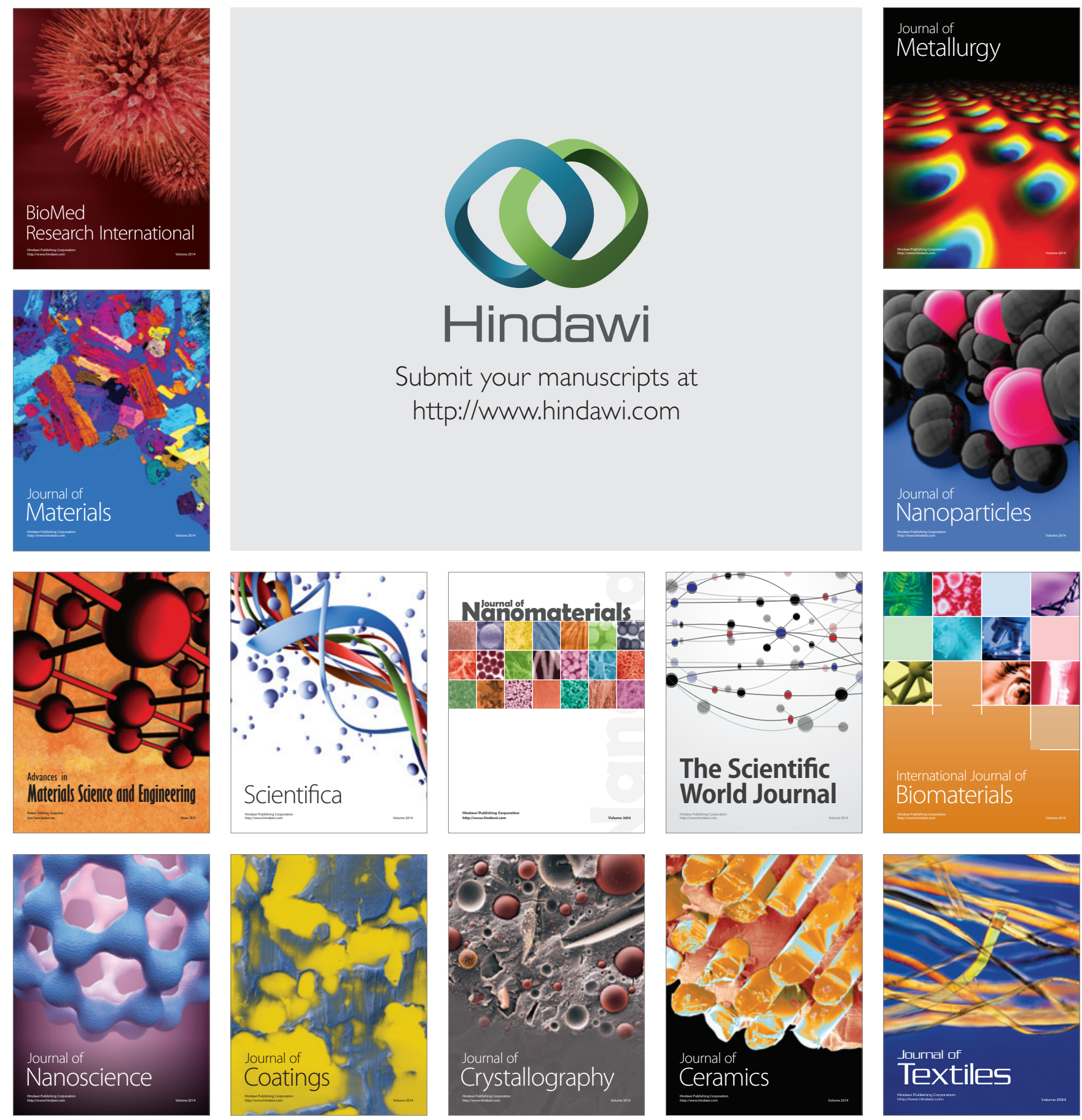